\title{
THE MAGNETISM OF AMORPHOUS METALS AND ALLOYS
}


This page is intentionally left blank 


\section{THE MAGNETISM OF AMORPHOUS METALS AND ALLOYS}

Editors

J. A. Fernandez-Baca

Oak Ridge National Laboratory, USA

Wai-Yim Ching

University of Missouri - Kansas City, USA 
Published by

World Scientific Publishing Co. Pte. Ltd.

P O Box 128, Farrer Road, Singapore 9128

USA office: Suite 1B, 1060 Main Street, River Edge, NJ 07661

UK office: 57 Shelton Street, Covent Garden, London WC2H 9HE

\section{THE MAGNETISM OF AMORPHOUS METALS AND ALLOYS}

Copyright $\odot 1995$ by World Scientific Publishing Co. Pte. Ltd.

All rights reserved. This book, or parts thereof, may not be reproduced in any form or by any means, electronic or mechanical, including photocopying, recording or any information storage and retrieval system now known or to be invented, without written permission from the Publisher.

For photocopying of material in this volume, please pay a copying fee through the Copyright Clearance Center, Inc., 222 Rosewood Drive, Danvers, Massachusetts 01923, USA.

ISBN 981-02-1033-7

Printed in Singapore. 


\section{CONTENTS}

Foreword $\quad$ xi

\section{THEORY OF MAGNETISM IN AMORPHOUS TRANSITION} METALS AND ALLOYS

by Yoshiro Kakehashi and Hiroshi Tanka

1. Introduction 1

2. Theoretical Approach to Amorphous Metallic Magnetism 3

2.1. Basic problems and developments 3

2.1.1. Amorphous structure 4

2.1.2. Electronic structure calculations $\quad 7$

2.1.3. Calculating magnetic properties $\quad 8$

2.2. LMTO-recursion method 9

2.2.1. Muffin-tin method and linear method 9

2.2.2. Tight-binding LMTO method 11

$\begin{array}{ll}\text { 2.2.3. Recursion method } & 17\end{array}$

2.3. Finite-temperature theory of amorphous metallic magnetism 19

2.3.1. Functional integral method 19

2.3.2. Local moment in an effective medium 22

2.3.3. Structural disorder and electronic states 24

2.3.4. Distribution-function method 26

2.3.5. Extension to amorphous alloys $\quad 29$

3. Magnetic Properties of Amorphous Transition Metals 32

3.1. General survey $\quad 32$

3.2. Amorphous $\mathrm{Fe} \quad 37$

3.3. Amorphous $\mathrm{Co}$ and $\mathrm{Ni} \quad 44$

4. TM Amorphous Alloys $\quad 48$

4.1. TM-TM amorphous alloys $\quad 48$

4.1.1. General aspects 48

4.1.2. $\mathrm{Fe}-\mathrm{Zr}$ amorphous alloys $\quad 50$

4.1.3. Fe-Ni amorphous alloys $\quad 52$

4.1.4. Co-Y amorphous alloys $\quad 54$

4.2. RE-TM amorphous alloys 56

4.2.1. General aspects 56 
4.2.2. Gd-Fe alloys $\quad 59$

4.2.3. Gd-Co alloys 63

4.3. TM-metalloid alloys $\quad 67$

4.3.1. General aspects 67

$\begin{array}{ll}\text { 4.3.2. Fe-B alloys } & 70\end{array}$

$\begin{array}{ll}\text { 4.3.3. Co-B alloys } & 72\end{array}$

References $\quad 76$

2. ELECTRONIC STRUCTURE CALCULATIONS IN MAGNETIC METTALIC GLASSES

by W.Y. Ching

1. Introduction $\quad 85$

2. The Orthogonalized Linear Combination of Atomic Orbitals Method 87

2.1. The atomic basis functions $\quad 87$

2.2. The site-decomposed potential functions 88

2.3. The technique of gaussian transformation 91

2.4. The technique of core-orthogonalization 96

2.5. The secular equation 98

2.6. Spin-polarized calculations $\quad 99$

2.7. Scalar relativistic correction $\quad 100$

2.8. Spin-orbit coupling 102

2.9. Calculation of physical observables 104

3. Application to Metallic Glasses 109

3.1. Periodic structure models 109

3.2. Amorphous $\mathrm{Ni}$ and $\mathrm{Ni}-\mathrm{P}$ glasses 112

3.3. Amorphous $\mathrm{Cu}-\mathrm{Zr}$ glasses $\quad 114$

3.4. Amorphous $\mathrm{Mg}-\mathrm{Zn}$ glasses $\quad 116$

3.5. Transport properties 117

3.6. Optical properties 123

4. Application to Magnetic Glasses 125

4.1. Crystalline $\mathrm{FeB}, \mathrm{Fe}_{2} \mathrm{~B}$ and $\mathrm{Fe}_{3} \mathrm{~B}$ compounds 125

4.2. Amorphous $\mathrm{Fe}$ model 127

4.3. Amorphous $\mathrm{Fe}_{80} \mathrm{~B}_{20}$ model 132

5. Some Conclusions and Future Directions 136 
Acknowledgements $\quad 137$

$\begin{array}{ll}\text { 6. References } & 137\end{array}$

3. RANDOM ANISOTROPY IN AMORPHOUS ALLOYS

by Eugene M. Chudnovsky

1. The Random Anisotropy Model 143

2. The Correlated Spin Glass 150

2.1. The Imry-Ma arguments 150

2.2. The random-anisotropy XY model 152

2.3. The random-anisotropy mean-spherical model 155

2.4. Experimental evidence of the correlated spin glass 157

2.5. The phase diagram 159

2.6. Coherent anisotropy 160

3. Behavior in a Magnetic Field 162

3.1. Zero-field susceptibility of the CSG 163

3.2. Magnetic disorder in the presence of a field 166

3.3. The magnetization curve 168

3.4. Dimensional crossover 171

Acknowledgements $\quad 173$

$\begin{array}{ll}\text { References } & 173\end{array}$

4. MAGNETIC PROPERTIES OF VAPOR-QUENCHED AMORPHOUS AND METASTABLE CRYSTALLINE ALLOYS by C.L. Chien

1. Introduction 175

2. Rapid Quenching Processes and Vapor Quenching Methods 178

3. Characterizations and Measurements of Magnetic Properties 180

4. Magnetic Properties of Crystalline Fe and Co 181

5. Composition Ranges for Amorphous Alloys 183

6. Magnetic Properties of TM-Metalloid Systems 185

7. Magnetic properties of TM-ET Systems 194

8. Toward the Amorphous Pure Metal Limit 202

9. Metastable Crystalline Systems 204 
viii

10. Conclusions $\quad 212$

$\begin{array}{ll}\text { Acknowledgements } & 214\end{array}$

$\begin{array}{ll}\text { References } & 215\end{array}$

5. NEUTRON SCATTERING STUDIES OF THE SPIN DYNAMICS

OF AMORPHOUS ALLOYS

by Jeffrey $W$. Lynn and Jamie A. Femandez-Baca

1. Introduction

221

2. Neutron Scattering $\quad 223$

2.1. Unpolarized neutron scattering cross sections 223

2.2. Polarized neutron cross sections 226

2.3. Neutron scattering from amorphous alloys 229

3. Spin Dynamics of Isotropic Ferromagnets 231

3.1. Small q 231

3.2. Invar systems 239

3.3. Large $q$ excitations $\quad 243$

4. Spin Dynamics of Amorphous Frustrated Magnets 246

4.1. Diluted magnets $\quad 247$

4.2. Iron-rich systems $\quad 249$

5. Future Directions 254

Acknowledgements $\quad 255$

References $\quad 255$

6. NUMERICAL STUDIES OF MAGNONS IN AMORPHOUS MAGNETS by D. L.Huber

1. Introduction $\quad 261$

1.1. Overview $\quad 261$

1.2. Magnons $\quad 262$

1.3. Eigenvalues and eigenvectors $\quad 263$

1.4. Magnetic materials and model Hamiltonians 264

2. Amorphous Ferromagnets 265

2.1. Overview 265

2.2. Calculations 266 
2.3. Discussion 268

3. Ferromagnets with Random Axis Anisotropy 269

3.1. Overview 269

3.2. Calculations 270

3.3. Discussion 273

4. Summary and Conclusions 276

5. References 276

6. Appendix 277

6.1. Overview 277

6.2. Recursion relations 277

6.3. Equation-of-motion method 279

7. MAGNETISM AND MAGNETO-OPTICS OF RARE EARTH-TRANSITION METAL GLASSES AND MULTLAYERS

by D. J. Sellmyer, R.D. Kirby and S. S. Jaswal

1. Introduction and Scope 281

2. Overview of Magnetic Interactions and Structures 283

3. Perpendicular Anisotropy in Films and Multilayers 288

4. Electronic Structure and Magneto-Optic Effects 298

5. Domain Dynamics and Magnetization Reversal 305

5.1. Experimental results 307

5.1.1. Direct observations of magnetic domains 307

5.1.2. Time dependence of remanence decay and mgnetization reversal 311

5.2. Models of magnetization reversal 315

5.2.1. Street and Woolley model 315

5.2.2. Single barrier height approach $\quad 317$

5.2.3. The Fatuzzo model $\quad 320$

5.2.4. Micromagnetic calculations and simulations 324

6. Concluding Remarks $\quad 330$

Acknowledgements $\quad 332$

References $\quad 332$ 
This page is intentionally left blank 


\title{
FOREWORD
}

About four years ago we were invited by the editors of World Scientific Publishing Co. Pte. Ltd. to eclit a review volume on selected topics of randomness in magnetism. This subject was later narrowed down to the magnetism of amorphous metallic alloys. Of course the first question that came to our minds is "Why bother in editing a volume on a subject that has already excellent and comprehensive review books and articles like those of Moorjani and Coey ${ }^{1}$ and J. Chappert. ${ }^{2}$ ?" The immediate answer was that perhaps it was worthwhile to make an update to the literature in this field, in the form of a book that would gather a selection of topics written by the people that had made the most recent progress. This would offer current reviews from the point of view of the specialists who would concentrate on their most recent progress while not neglecting to refer the reader to the already abundant existing literature. With this in mind we invited a selected group of specialists to review their progress in this subject, some of them accepted this challenge and this book is the result of their hard work. We thank all of them for their dedication and for their commitment. Because of the way that this book came about, this volume is by no means a comprehensive review of all the progress in the subject. Rather it is a collection of selected topics in the recent progress on the study of the magnetism of amorphous metals and alloys. Finally, we recognize the title of this book is very ambitious because while a great deal has been learned about the magnetism of amorphous metallic alloys, the study of the magnetism of the pure amorphous metals is still a subject of intense activity. We hope that the content of this book will help to motivate research in this direction. We trust that the reader will find in this volume a worthwhile update of some of the progress in the field of amorphous magnetism.

\author{
J. A. Fernandez-Baca \\ W. Y. Ching
}

${ }^{1}$ K. Moorjani and J. M. D. Coey, Magnetic Glasses, (Elsevier, 1984).

${ }^{2}$ J. Chappert, "Magnetism of Amorphous Metallic Alloys", in Magnetism of Metals and Alloys, ed. M. Cyrot (North Holland, 1982) 